Revue des sciences de l'eau

REVUEDES

Journal of Water Science

S C I E N CES

DE L'EA U

\title{
Modélisation du transport des solutés neutres à travers des membranes de nanofiltration Modelisation of transport of neutral solutes through nanofiltration membranes
}

\author{
F. Boucard et A. Saboni
}

Volume 13, numéro 4, 2000

URI : https://id.erudit.org/iderudit/705400ar

DOI : https://doi.org/10.7202/705400ar

Aller au sommaire du numéro

Éditeur(s)

Université du Québec - INRS-Eau, Terre et Environnement (INRS-ETE)

ISSN

0992-7158 (imprimé)

1718-8598 (numérique)

Découvrir la revue

Citer cet article

Boucard, F. \& Saboni, A. (2000). Modélisation du transport des solutés neutres à travers des membranes de nanofiltration. Revue des sciences de l'eau / Journal of Water Science, 13(4), 405-419. https://doi.org/10.7202/705400ar
Résumé de l'article

L'exclusion engendrée par la présence de membranes de nanofiltration est due à la superposition de plusieurs mécanismes. Aussi, il est important de pouvoir distinguer ces différents modes de transports afin de les comprendre et de proposer des modèles adéquats. Cette étude s'attachera uniquement à la compréhension du transport de solutés neutres à travers des membranes de ce type. Trois sucres, le glucose, le saccharose et le raffinose, ont été utilisés pour caractériser deux membranes organiques fournies par la société Osmonics. Ce travail montre qu'un modèle simple, basé sur la diffusion, tenant compte des conditions hydrodynamiques du module, est en accord avec les résultats obtenus au laboratoire mais également trouvés dans la littérature. Une bonne adéquation entre le modèle et l'expérience est ainsi obtenue, à la fois sur des modules plans et tubulaires, pour des écoulements laminaires et turbulents. 


\title{
Modélisation du transport des solutés neutres à travers des membranes de nanofiltration
}

\section{Modelisation of transport of neutral solutes through nanofiltration membranes}

\author{
F. BOUCARD, A. SABONI
}

Reçu le 5 octobre 1999, accepté le 6 novembre $2000^{\star \star}$.

\section{SUMMARY}

\begin{abstract}
Although nanofiltration appeared at the end of the 1970s under various names, it was only really recognized as a useful separation process in the 1980s. Nanofiltration membranes are porous media with a mean pore diameter around 1 nanometer. These membranes do not obey the traditional solution-diffusion model given for reverse osmosis or the convection-diffusion model used to describe ultrafiltration. Although the technique has benefited from a fast technological development, the transport mechanisms are still misunderstood and for a particular separation the choice of a nanofiltration membrane remains empirical.
\end{abstract}

The main objective of this work was to understand and to model the transport of neutral solutes through nanofiltration membranes. Neutral solutes were chosen to emphasise geometrical exclusions, to avoid any electrical interactions and to identify the preponderant transport mechanisms through these materials. The experiments were carried out with a laboratory filtration apparatus. The membranes were laid out in a parallel plane osmotic cell, which makes tangential filtration possible. The geometry of the filtration cell involved the choice of two organic membranes supplied as flat sheets: a BQ01 and a MX07 membrane. The filtration area was $86 \mathrm{~cm}^{2}$. The pressure varied from 7 to 30 bars. The temperature was maintained at $20^{\circ} \mathrm{C}$ whereas tangential velocity in the cell was fixed at $0.45 \mathrm{~m} \cdot \mathrm{s}^{-1}$ (the Reynolds number was 3350). As the solutions used were slightly concentrated, the pH remained close to neutral pH. Three sugars were chosen as solutes: glucose, saccharose and raffinose. These molecules have two advantages: they are electrically neutral and they have molecular weights close to the membranes'MWCO, as provided by the manufacturer.

First, saccharose was studied on the two membranes with two different concentrations. These experiments showed that the separation of neutral

Laboratoire de Thermodynamique des Procédés, Université de Caen, IUT, Département GTE, 120 rue de l'Exode, 50000 Saint-Lô, France

* Correspondance. E-mail : Itp@stlo.unicaen.fr

** Les commentaires seront reçus jusqu'au 14 septembre 2001. 
solutes by nanofiltration membranes is due only to a sieving effect. In subsequent experiments a single concentration was used to characterize the retentions of both glucose and raffinose. The results of the filtrations carried out on the three sugars validated the molecular weight cut-off specified by the manufacturer: the MWCO of the BQ01 membrane was estimated to be $1000 \mathrm{Da}$, and that for the MX07 membrane was estimated as $200 \mathrm{Da}$.

Schematically, the solute transport can be divided into three stages: in the feed, at the feed/membrane interface, and within the membrane material. In the feed, one notes an increase in solute concentration if one approaches the membrane from upstream. This phenomenon, which is general to any selective transport, is called concentration polarization and is described by film theory. This theory stipulates the creation of an antagonistic diffusive flow, from the membrane towards the feed, seeking to restore the concentration balance within the feed solution. The modification of the concentration at the feed/membrane interface leads to the definition of two retention coefficients: a measured value, the observed retention $\left(R_{\text {obs }}\right)$, and a calculated value, the intrinsic retention $\left(\mathbf{R}_{\mathrm{m}}\right)$. Steric exclusion based on the size difference between the pore and the solute is set up at the interface. Uncharged solutes can be visualised as rigid spheres and the membranes can be regarded as a bundle of cylindrical, parallel, rigid and right capillaries. Since the elements are rigid and the solutes are subjected to the same geometrical constraints at the entry and at the exit of a pore, the partition coefficients are identical at those two ends. Finally, lying between reverse osmosis and ultrafiltration, transport through nanofiltration membranes is often expressed as the sum of convective and diffusive phenomena. However, the experimental results show that the observed retentions are stable or increase when pressure increases. These observations also highlight the fact that the values of infinite retention are always compatible with values close to 1 . These observations corroborate the idea that diffusion is the predominant transport mechanism of neutral species through the studied materials (BQ01, MX07). The transport equation of neutral solutes can then be simplified to its diffusive component. The expression of the intrinsic retention is obtained by using Fick's law, the definition of the retention coefficients and the definition of the partition coefficients:

$$
R_{m}=1-\frac{1}{1+J_{v} \alpha}
$$

The geometrical and physicochemical characteristics of the solutes and of the membranes merge into the $\alpha$ parameter.

The results found with the theoretical relation were confronted with experimental data derived from film theory (in order to take into account concentration polarization). The simple one-parameter model was successful in correlating the results obtained in this work. The model was also tested with data coming from COMBE $e t$ al. (1997), who studied filtrations of glucose, saccharose and raffinose in a laminar flow system by ceramic nanofiltration membranes laid out in the shape of tubular module. The results obtained show that the simple model also successfully correlates with the performances of these membranes.

With the data obtained in our laboratory as well with the data found in literature, this study shows that a simple one-parameter model, based on the diffusional transport of the solutes within the membrane material, predicts the rejection of neutral solutes by nanofiltration membranes. The simple oneparameter model is able to simulate any filtration carried out by these membranes for different circulation conditions, for diverse geometrical shapes and for various materials.

Keywords: nanofiltration, neutral solutes, diffusive model, concentration polarization. 


\section{RÉSUMÉ}

L'exclusion engendrée par la présence de membranes de nanofiltration est due à la superposition de plusieurs mécanismes. Aussi, il est important de pouvoir distinguer ces différents modes de transports afin de les comprendre et de proposer des modèles adéquats. Cette étude s'attachera uniquement à la compréhension du transport de solutés neutres à travers des membranes de ce type. Trois sucres, le glucose, le saccharose et le raffinose, ont été utilisés pour caractériser deux membranes organiques fournies par la société Osmonics. Ce travail montre qu'un modèle simple, basé sur la diffusion, tenant compte des conditions hydrodynamiques du module, est en accord avec les résultats obtenus au laboratoire mais également trouvés dans la littérature. Une bonne adéquation entre le modèle et l'expérience est ainsi obtenue, à la fois sur des modules plans et tubulaires, pour des écoulements laminaires et turbulents.

Mots clés : nanofiltration, solutés neutres, modèle diffusif, polarisation de concentration.

\section{1 - INTRODUCTION}

Apparue à la fin des années soixante-dix sous diverses appellations, la nanofiltration n'a véritablement été reconnue comme technique séparatrice à part entière qu'au cours des années quatre-vingt. Situées entre l'osmose inverse et l'ultrafiltration, les membranes de nanofiltration sont utilisées dans une gamme de pression comprise entre 10 et 40 bars alors que leur seuil de coupure est généralement situé entre 200 et $1000 \mathrm{Da}$. Assurant des flux supérieurs à l'osmose inverse tout en gardant des capacités de rétention importantes notamment grâce à la présence de charges électriques dans la couche active, l'engouement pour ces nouveaux matériaux a été immédiat. Ainsi, la nanofiltration est aujourd'hui implantée dans l'industrie laitière (TIMMER et al., 1994) et fait son apparition dans l'industrie sucrière (WADLEY et al., 1995) ou encore papetière (BERTRAN et al., 1997). Bien que profitant d'un développement technologique rapide, il reste cependant que ses mécanismes de transport et de séparation ne sont pas totalement éclaircis (TSURU et al.,1999; YAROSHCHUCK, 1999). En effet, disposant de pores de l'ordre de $1 \mathrm{~nm}$ de diamètre, ces membranes ne répondent plus au schéma classique de solubilisation-diffusion posé pour l'osmose inverse ou de convection-diffusion décrivant l'ultrafiltration.

Afin d'éviter toute définition du matériau, certains auteurs (YAROSHCHUCK et VOVKOGON, 1994 ; BATICLE et al., 1997 ; MEHIGUENE et al., 1999) considèrent la membrane comme une «boite noire » et modélisent le transport membranaire selon la thermodynamique irréversible (SPIEGLER et KEDEM, 1966). Le côté intermédiaire de la nanofiltration entre l'ultrafiltration et l'osmose inverse a également amené de nombreux auteurs à supposer ces transferts régis à la fois par la convection et par la diffusion (COMBE et al., 1997 ; SARRADE et al., 1994 ; BOWEN et al., 1997). Lors d'études menées sur des membranes organominérales, RIOS et al. (1996) affirment quant à eux, que la diffusion est négligeable et que les transferts sont essentiellement convectifs. Partant de leurs observations expérimentales, LEVENSTEIN et al. (1996) arrivent à une constatation dia- 
métralement opposée et supposent que la diffusion est le mode de transport majoritaire en nanofiltration. Cependant, bien que disposant de plusieurs approches théoriques, le choix d'une membrane de nanofiltration pour une utilisation précise reste aujourd'hui tributaire de l'expérience.

Cette étude s'attachera à comprendre et à modéliser le transport de solutés neutres à travers deux membranes distinctes afin de mieux appréhender certains mécanismes d'exclusion. Les interactions électriques seront abordées dans un travail ultérieur. À l'aide des résultats obtenus au laboratoire mais également trouvés dans la littérature, ce travail montre qu'un modèle simple, basé sur le transport diffusionnel des espèces dans la membrane et tenant compte des conditions hydrodynamiques du module, prédit parfaitement les performances du matériau.

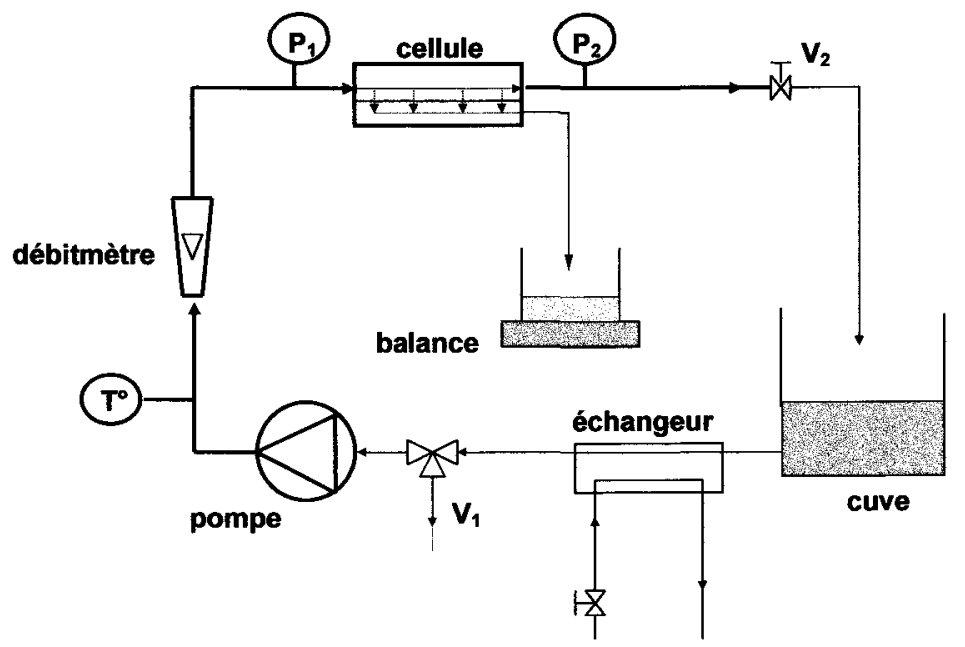

Figure 1 Schéma du pilote expérimental.

Experimental setup.

\section{2 - DESCRIPTION DES EXPÉRIENCES}

\subsection{Dispositif}

Les expériences ont été réalisées sur un pilote de laboratoire (figure 1). La pression, assurée par l'intermédiaire d'une pompe volumétrique, était ajustée par une vanne pointeau $\left(V_{2}\right)$ et relevée à l'aide de deux manomètres $\left(P_{1}, P_{2}\right)$. La température du fluide était contrôlée par une sonde Pt100 tandis qu'un échangeur à eau perdue en assurait sa régulation. Le débit d'alimentation était mesuré grâce à un débitmètre à hélice alors que le débit de perméat était obtenu par empotage chronométré.

Schématisée par la figure 2 , la cellule plane réalisée au laboratoire a été mise en place afin d'effectuer des filtrations tangentielles : le fluide était amené côté rétentat par le capot d'alimentation supérieur (2) et arrivait tangentielle- 


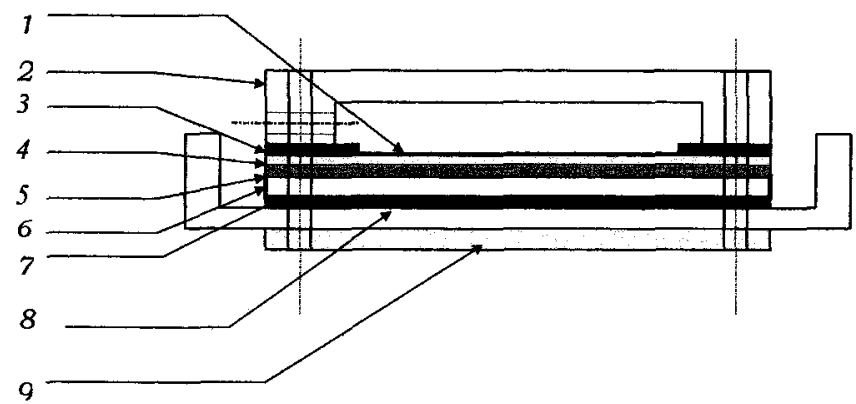

Figure 2 Vue en coupe de la cellule de filtration à flux tangentiel.

Side view of the experimental tangential cell.

ment en contact avec la membrane (1). Celle-ci était supportée par un empilement de tissu métallique (4), de plaques métallique et plastique (5 et 6 ). Les étanchéités étaient assurées par divers joints plats $(3,7)$ alors que le perméat était collecté dans le bac de récupération (8). Une plaque en acier (9) assurait la tenue mécanique de l'ensemble.

La géométrie du module de filtration a entraîné un choix parmi des membranes planes disponibles sur le marché. Les deux matériaux retenus ont été des membranes organiques fournies par la société Osmonics. La surface de filtration était de $86 \mathrm{~cm}^{2}$. Les propriétés de ces matériaux, référencés BQ01 et MX07, sont regroupées dans le tableau 1. Les membranes étaient stockées dans de l'eau déminéralisée durant 48 heures puis compactées sous 20 bars pendant plusieurs heures avant toute première utilisation. Ces opérations avaient pour but de s'affranchir de problèmes éventuels de gonflement mais aussi d'obtenir un matériau stable structurellement (ROSA et PINHO, 1994).

Tableau 1 Caractéristiques des membranes données par le fabricant (Osmonics).

Table 1 Characteristics of the membranes given by the manufacturer (Osmonics).

\begin{tabular}{|c|c|c|c|}
\hline Rétérence & Matériau & Rétention du NaCl (\%) & MWCO (Da) \\
\hline MX07 & polyamide & $50-70$ & $300-600$ \\
BQ01 & polymère X & $20-30$ & $400-900$ \\
\hline
\end{tabular}

\subsection{Solutés}

Afin d'obtenir des rétentions significatives et de préciser les seuils de coupure des échantillons utilisés, les solutés choisis devaient disposer de poids moléculaires voisins des MWCO communiqués par le fabricant (tableau 1). Une série de trois sucres a été retenue : le glucose, le saccharose et le raffinose. Fournis par la société Fisher Scientific, ces éléments étaient pur au minimum à $99,9 \%$. Leurs principales caractéristiques sont regroupées dans le tableau 2. 
Tableau 2 Caractéristiques des sucres $\left(r_{\mathrm{s}}\right.$ calculés à partir de la relation de Stokes-Einstein).

Table 2 Characteristics of sugars $\left(r_{s}\right.$ calculated starting from the Stokes-Einstein relation).

\begin{tabular}{|c|c|c|c|}
\hline Sucre & $\mathbf{M}(\mathbf{D a})$ & $\mathbf{D}_{\infty}\left(\mathbf{m}^{2} \cdot \mathbf{s}^{-\mathbf{1}}\right)$ & Rayon de Stokes, $\mathbf{r}_{\mathbf{s}}(\mathbf{m})$ \\
\hline Glucose & 180,16 & $6,9 \times 10^{-10}$ & $3,2 \times 10^{-10}$ \\
Saccharose & 342,3 & $5,2 \times 10^{-10}$ & $4,2 \times 10^{-10}$ \\
Raffinose & 504,52 & $4,2 \times 10^{-10}$ & $5,2 \times 10^{-10}$ \\
\hline
\end{tabular}

Les coefficients de diffusions sont les valeurs citées par COMBE et al. (1997) d'après les travaux de NAKAO et KIMURA (1981) et les rayons de Stokes sont calculés à l'aide de la relation de Stokes-Einstein.

\subsection{Démarche expérimentale}

Seule la pression (de 7 à 30 bars) et la concentration de l'alimentation ont été envisagées en tant que variables. Les autres paramètres étaient préalablement fixés et restaient constants durant l'ensemble des manipulations. Ainsi, la température était régulée à $20 \pm 1{ }^{\circ} \mathrm{C}$ tandis que le débit de circulation était maintenu à $5,13 \mathrm{I} \cdot \mathrm{mn}^{-1}$, soit une vitesse tangentielle dans la cellule de $0,45 \mathrm{~m} \cdot \mathrm{s}^{-1}$ et un nombre de Reynolds de 3350 . Les solutions utilisées étant des solutions faiblement concentrées, le $\mathrm{pH}$ restait toujours proche du $\mathrm{pH}$ neutre.

Après chaque expérience, l'installation était entièrement nettoyée. Ce nettoyage impliquait à la fois le rinçage du réseau hydraulique, mais aussi le décolmatage de la membrane. II s'effectuait à l'aide d'une solution à base de soude ( $\mathrm{pH} 11$ à 12) selon les indications données par le fabricant. On vérifiait ensuite que le flux original de la membrane était bien retrouvé. Dans le cas contraire on procédait à un nouveau nettoyage, voire à un remplacement de la membrane.

Le saccharose a tout d'abord été étudié sur les deux matériaux à deux concentrations distinctes : $10^{-1} \mathrm{~mol} \cdot \mathrm{I}^{-1}$ et $5 \times 10^{-2} \mathrm{~mol} \cdot \mathrm{I}^{-1}$. Une unique concentration a ensuite été employée pour caractériser la rétention des autres sucres, soit $10^{-1} \mathrm{~mol} \cdot \mathrm{I}^{-1}$ pour le glucose et $6,5 \times 10^{-3} \mathrm{~mol} \cdot \mathrm{I}^{-1}$ pour le raffinose. Les concentrations étaient mesurées à l'aide d'un réfractomètre (Atago DR-A1).

\section{3 - APPROCHE THÉORIQUE}

\subsection{Hypothèses}

Les membranes de nanofiltration sont des milieux poreux dont la taille moyenne des pores est de l'ordre du nanomètre. Afin d'avoir une représentation spatiale, les solutés sont apparentés à des sphères rigides et les pores 
sont supposés cylindriques, rigides et droits, autorisant à parler de diamètre de pores. On considère également que le rapport de la longueur du pore sur son diamètre est très grand. II est alors aisé à travers cette représentation, de concevoir une exclusion stérique basé sur la différence de taille entre le pore et le soluté. Les éléments étant rigides et les solutés étant soumis aux mêmes contraintes géométriques en entrée et en sortie de pore, les coefficients de partage sont identiques à ces deux extrémités. Ils sont donnés par :

$$
\begin{gathered}
\Phi_{\text {entrée }}=\Phi_{\text {sortie }}=\Phi_{s} \\
\bar{C}_{s}(0)=\Phi_{s} C_{m} \\
\bar{C}_{s}(L)=\Phi_{s} C_{p}
\end{gathered}
$$

La nanofiltration étant intermédiaire entre l'ultrafiltration et l'osmose inverse, il est important de savoir si ces transferts de matière sont régit par la convection, par la diffusion, voire par les deux simultanément :

- pour la membrane BQ01, les rétentions observées sont constantes quelque soit le soluté et la pression transmembranaire appliquée (figure 4);

- pour la membrane MX07, la figure 5 montre une légère croissance de rétention observée du glucose en fonction de la pression;

- la figure 6 montre que la rétention infinie du glucose est supérieure à 0,8 , que celle du raffinose tend graphiquement vers 1 tout comme la rétention infinie du glucose filtré par la membrane MX07 (figure 7);

- du fait de la limite mécanique du matériau, il est impossible de donner graphiquement une valeur pour la rétention infinie du glucose filtré par la membrane BQ01 (figure 6).

L'association d'une croissance ou d'une stabilité de la rétention observée en fonction de la pression avec des rétentions infinies compatibles avec 1 , suppose que la diffusion joue un rôle majeur dans le transport des espèces pour ces deux matériaux (VAN DER HORST et al., 1995).

Par ailleurs, il semble difficile de pouvoir adopter un coefficient de diffusion identique dans la solution d'alimentation et à l'intérieur de la membrane. En effet, les solutés se trouvant dans un espace réduit lors du transfert dans la membrane, l'environnement et les interactions se trouvent modifiés, conduisant à la définition d'une diffusivité interne du soluté $\left(D_{s}\right)$. En outre, du fait d'un ordre de grandeur voisin entre les solutés et le diamètre moyen de pore, il ne peut exister en nanofiltration que des gradients selon l'épaisseur de la membrane. Toute représentation de gradient (de vitesse, de concentration...) selon le rayon du pore est ici physiquement inacceptable.

\subsection{Modélisation}

\subsubsection{Polarisation de concentration}

Lorsque depuis l'amont on se rapproche de la membrane, on constate une augmentation de la concentration (figure 3). Ce phénomène appelé polarisation de concentration et qui est général à tout transport sélectif, peut être décrit par la théorie du film. Cette théorie stipule alors la création d'un flux antagoniste, 


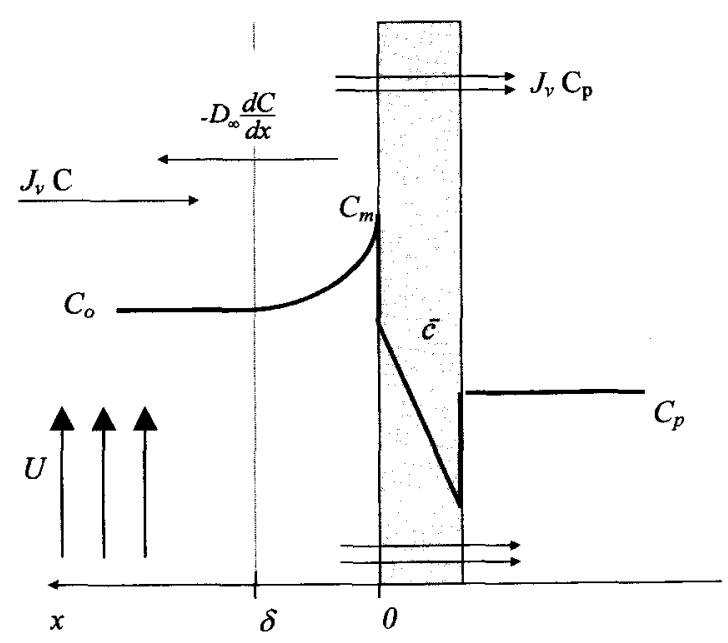

Figure 3 Polarisation de concentration.

Concentration polarization.

de la membrane vers l'alimentation, cherchant à rétablir l'équilibre au sein du milieu. Le bilan de matière relatif à l'espèce retenue permet d'écrire :

$$
J_{\nu} C_{p}=J_{\nu} C-D_{\infty} \frac{d C}{d x}
$$

Les conditions aux limites sont données par:

$$
\begin{array}{ll}
x=\delta & C=C_{0} \\
x=0 & C=C_{m}
\end{array}
$$

L'intégration de l'équation (3) soumise aux conditions aux limites (4) permet ainsi de déterminer le flux du solvant :

$$
J_{v}=k \ln \left(\frac{C_{m}-C_{p}}{C_{o}-C_{p}}\right)
$$

avec : $k=\frac{D_{\infty}}{\delta}$,

où : $\quad k$ est le coefficient de transfert de matière ;

$D_{\infty}$ est le coefficient de diffusion dans l'alimentation ;

$\delta$ est l'épaisseur de la couche limite de concentration.

Cette épaisseur de la couche limite de concentration est fonction à la fois de la géométrie du module, du régime hydrodynamique, des propriétés physicochimiques du système et est calculée à l'aide du nombre de Sherwood. Dans le cas de cette étude, la section de passage du fluide est rectangulaire alors que la vitesse tangentielle d'écoulement est constante, fixée à $0,45 \mathrm{~m} \cdot \mathrm{s}^{-1}$. Le nombre de Reynolds étant alors de 3350 , le régime peut être considéré comme turbulent. Le nombre de Sherwood est alors calculé par (BRUN, 1988) :

$$
S h=0.04 \mathrm{Re}^{0.75} S c^{1 / 3}
$$




$$
\text { avec: } \operatorname{Re}=\frac{U \cdot d_{h}}{v}, \quad S c=\frac{v}{D_{\infty}} \quad \text { et : } S h=\frac{d_{h}}{\delta}
$$

Remplaçant Sh par son expression (6) dans l'équation (7), on en déduit $\delta$.

La modification de la concentration à l'interface membrane/alimentation a également comme conséquence d'amener à définir un nouveau taux de rétention. En effet, la membrane ne perçoit plus la concentration à l'infini mais cette nouvelle valeur. C'est pourquoi ce taux, appelé taux de rétention intrinsèque $\left(R_{m}\right)$, diffère du taux observé $\left(R_{o b s}\right)$ selon les relations suivantes :

$$
\begin{aligned}
& R_{\mathrm{obs}}=1-\frac{C_{p}}{C_{o}} \\
& R_{m}=1-\frac{C_{p}}{C_{m}}
\end{aligned}
$$

\subsubsection{Transfert dans la membrane}

Dans le cas de membranes de microfiltration et d'ultrafiltration, le flux de soluté est généralement exprimé selon la somme d'un terme convectif et d'un terme diffusif tel que :

$$
J_{s}=-D_{s} \frac{d \bar{c}_{s}}{d x}+\sqrt{C_{s}}
$$

Le côté intermédiaire de la nanofiltration vis-à-vis de l'osmose inverse et de l'ultrafiltration a longtemps fait que cette équation ou une de ses formes dérivées, ont été utilisées pour décrire les mécanismes de transfert pour ces membranes (COMBE et al., 1997 ; SARRADE et al., 1994 ; BOWEN et al., 1997). Lors de la position des hypothèses, on a supposé que la diffusion avait un rôle prépondérant dans le transport des espèces. On peut alors réduire l'équation de transport du soluté à :

$$
J_{s}=-D_{s} \frac{d \bar{c}_{s}}{d x}
$$

L'équation (10) est alors facilement intégrable sur la longueur $L$ du pore :

$$
J_{s}=J_{v} C_{p}=D_{s} \frac{\left[\bar{c}_{s}(0)-\bar{C}_{s}(L)\right]}{L}
$$

Les concentrations aux extrémités internes du pore de la membrane sont reliée aux concentrations extérieures par le coefficient de partage défini selon l'équation (2). Ainsi, à partir des relations (11), (2) et de la définition du taux de rétention (8.b), on aboutit à l'expression du taux de rétention intrinsèque :

$$
R_{m}=1-\frac{1}{1+J_{v} \alpha}
$$

avec : $\alpha=\frac{L}{D_{s} \Phi_{s}}$ 
D'autre part le taux de rétention intrinsèque peut être relié au taux de rétention observé par la relation suivante, dérivée de l'équation (5):

$$
R_{m}=\frac{1}{1+\left(\frac{1-R_{o b s}}{R_{o b s}}\right) \exp \left(-\frac{J_{v} \delta}{D_{\infty}}\right)}
$$

Connaissant les taux de rétention intrinsèques à partir de l'expérience (14), il est alors possible de comparer ces valeurs aux valeurs estimées issues du modèle théorique (12).

\section{4 - RÉSULTATS}

Lors de la filtration de solutés neutres, les membranes de nanofiltration n'ont théoriquement comme interaction que celle d'un tamisage classique où la concentration n'influe pas sur la rétention (COMBE et al., 1997). La séparation du saccharose par la membrane BQ01 à deux concentrations différentes confirme bien ce type de comportement (figure 4).

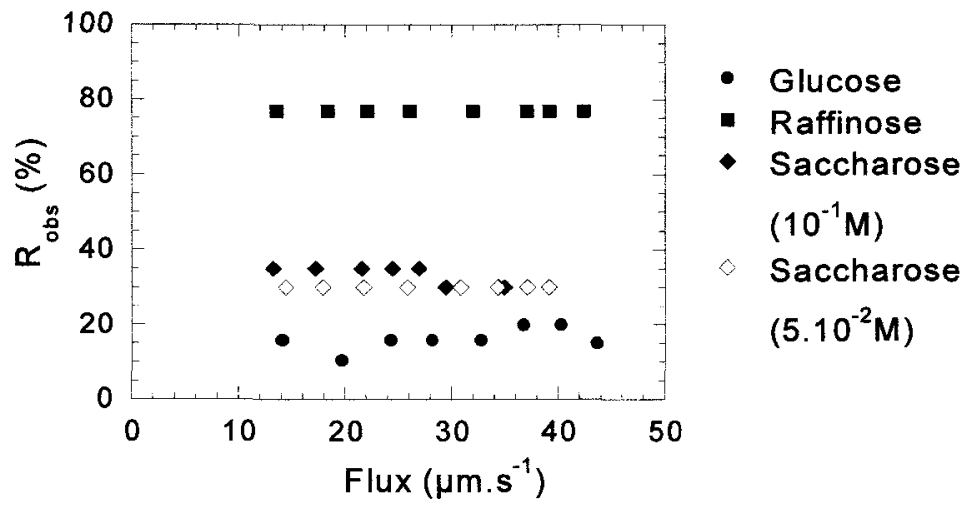

Figure 4 Taux de rétention observés pour la membrane BQ01.

Observed retention for the BQ01 membrane.

Le tableau 1 regroupe des indications statistiques constatées par le fabricant quant aux performances de ses matériaux, mais ne permet pas de connaître avec plus de précision le seuil de coupure (MWCO) ou les rétentions des échantillons fournis. À l'aide des figures 4 et 5 , le seuil de coupure de la membrane BQ01 est estimé à 1000 Da et celui de la membrane MX07 à $200 \mathrm{Da}$. La légère différence entre ces résultats expérimentaux et les valeurs fabricant (tableau 1) peut s'expliquer d'une part, par des méthodes de caractérisation différentes et d'autre part, par une variabilité quant à la reproductibilité de fabrication des matériaux (donc des performances). 


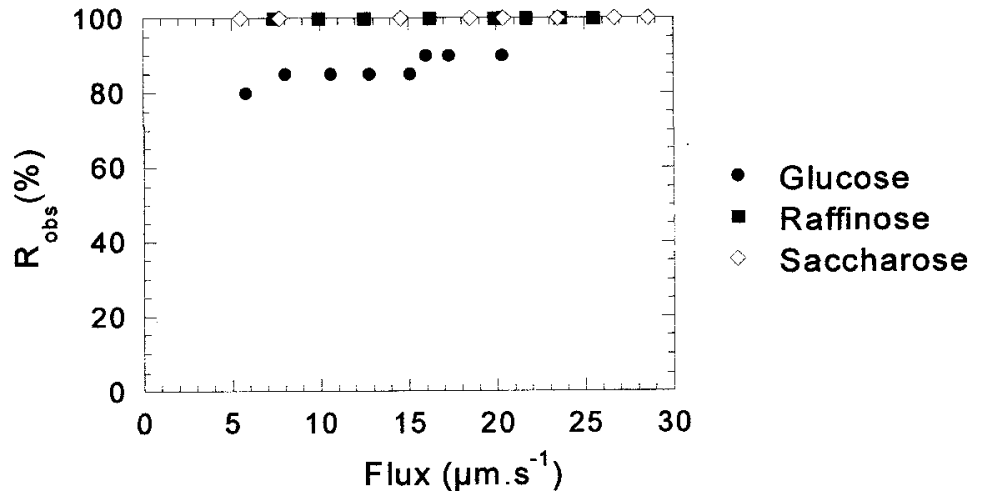

Figure 5 Taux de rétention observée pour la membrane MX07. Observed retention for the $M \times 07$ membrane.

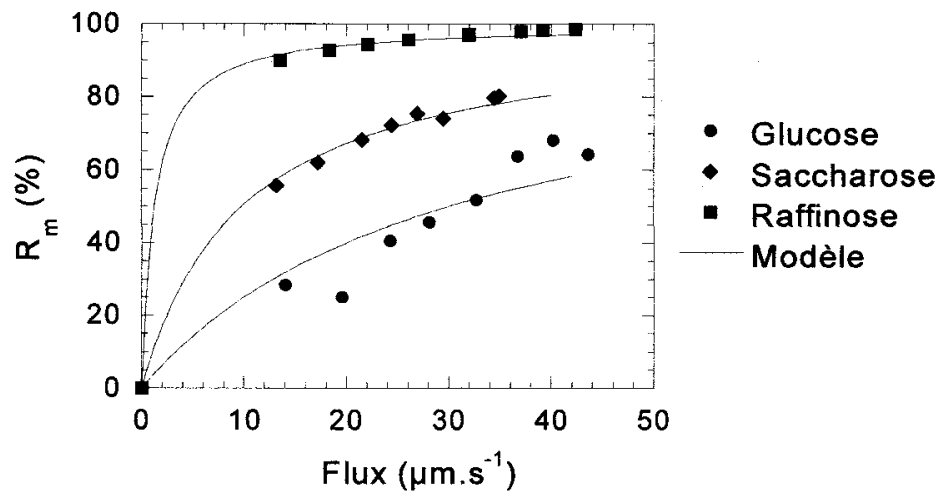

Figure 6 Taux de rétention intrinsèques expérimentaux et calculés pour trois sucres (membrane BQ01).

Experimental and calculated real retention for three sugars (BQ01 membrane).

Les résultats des modélisations effectuées à partir des expériences menées au laboratoire sont reportés sur les figures 6 et 7 . Ces graphes donnent les évolutions du taux de rétention intrinsèque expérimental calculé à partir de la théorie du film (14) et de la rétention observée, et du taux de rétention intrinsèque théorique estimé à partir du modèle simple (12). On note dans tous les cas une bonne corrélation entre le modèle et les résultats issus de l'expérience. Répertoriées dans le tableau 3, les valeurs du paramètre $\alpha$ sont obtenues à partir de l'équation (12) en procédant à une estimation par rapport aux résultats expérimentaux. Le paramètre $\alpha$ a pour principal avantage de regrouper l'ensemble des caractéristiques inconnues que sont le rayon moyen du pore $\left(r_{p}\right)$, sa longueur $(L)$ et la diffusivité dans le pore $\left(D_{S}\right)$. 


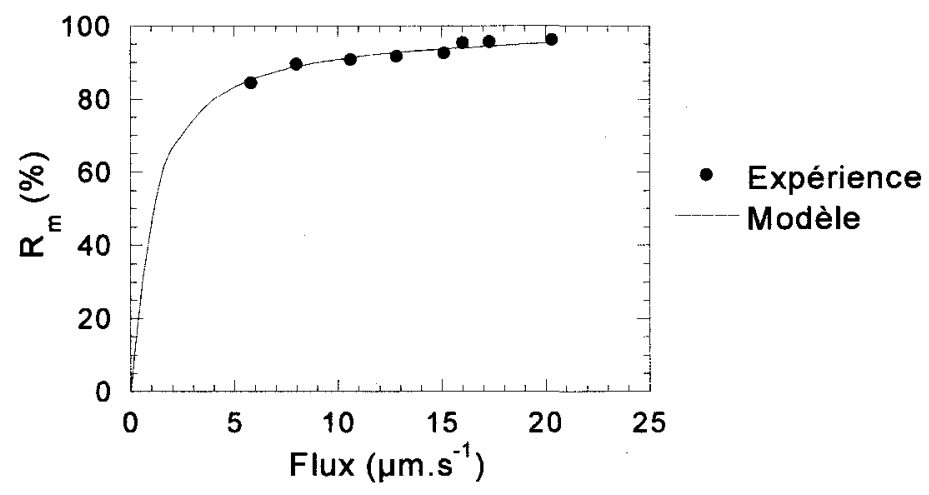

Figure 7 Taux de rétention intrinsèques expérimentaux et calculés pour le glucose (membrane MX07).

Experimental and calculated intrinsic retention for glucose ( $M \times 07$ membrane).

À partir de l'équation (13) et de deux valeurs estimées du paramètre $\alpha$, on peut approcher les caractéristiques structurelles $r_{p}$ et $L$ de la membrane, en s'appuyant sur les deux remarques suivantes :

- bien que ne connaissant pas les diffusivités des solutés dans le pore $\left(D_{\mathrm{s}}\right)$, on suppose en première approximation leur égalité avec les diffusivitès externes $D_{\infty}$ (tableau 2);

- le coefficient d'exclusion stérique est exprimé par la loi de Ferry :

$$
\Phi_{s}=\left(1-\frac{r_{s}}{r_{p}}\right)^{2}
$$

N'ayant qu'une valeur de $\alpha$ pour la membrane MX07, seule une approximation pour la membrane $\mathrm{BQ01}$ a pu être effectuée. Le diamètre moyen de pore est ainsi estimé à $1,09 \mathrm{~nm}$ tandis que la longueur moyenne du pore est estimée à $3,74 \mu \mathrm{m}$. II s'agit là de résultats cohérents pour une membrane de nanofiltration.

Des résultats issus de la littérature (COMBE et al., 1997) ont également été appréhendés afin de vérifier la validité de ce modèle sur une plus ample échelle. Il s'agit de valeurs obtenues en régime laminaire sur des membranes céramiques de nanofiltration disposées sous forme de modules tubulaires (Kerasep ${ }^{\mathrm{TM}}$, Tech-Sep). Les épaisseurs des couches de polarisation de concentration relatives aux trois sucres filtrés, sont respectivement de 75, 70 et $64 \mu \mathrm{m}$ pour le glucose, le saccharose et le raffinose. Connaissant ces données, il est possible de procéder au calcul de la rétention intrinsèque selon la relation (14) puis d'estimer celle-ci suivant l'équation (12). Les résultats de ces estimations sont reportés sur la figure 8. Les valeurs du paramètre $\alpha$ issu de la modélisation sont retranscrites dans le tableau 3. 


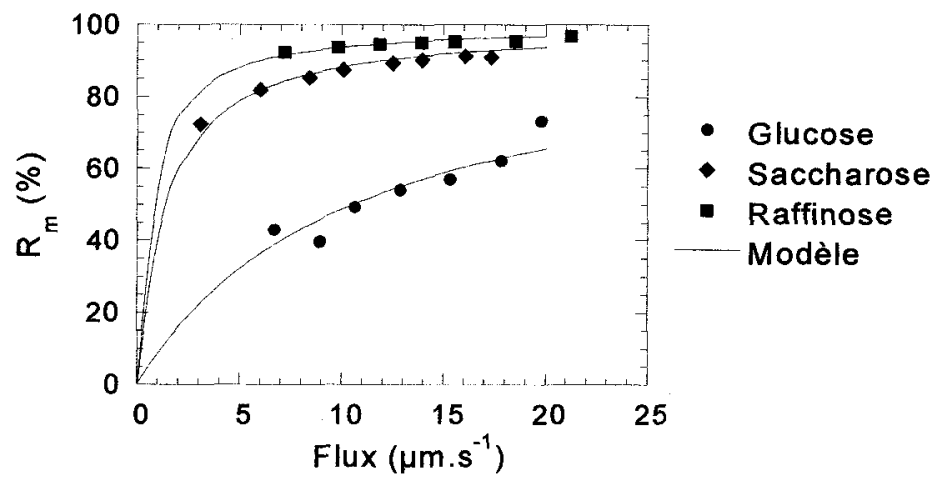

Figure 8 Taux de rétention intrinsèques expérimentaux (symboles) issus des travaux de COMBE et al. et estimés selon le modèle diffusif (courbes).

Experimental intrinsic retention (symbols) resulting from work of COMBE et al. and calculated according to the diffusive model (curves).

Tableau 3 Valeurs du paramètre $\alpha$ estimé numériquement $\left(\alpha=\frac{L}{D_{s} \Phi_{s}}\right)$.

Table 3 Values of the $\alpha$ parameter estimated numerically $\left(\alpha=\frac{\mathrm{L}}{\mathrm{D}_{\mathrm{s}} \Phi_{\mathrm{s}}}\right)$.

\begin{tabular}{|c|c|c|c|}
\hline & BQ01 & $\begin{array}{c}\alpha\left(s \cdot m^{-1}\right) \\
\text { MX07 }\end{array}$ & Kerasep $^{\top M}$ \\
\hline Glucose & 33386 & 999630 & 95084 \\
\hline Saccharose & 102540 & - & 743850 \\
\hline Raffinose & 809070 & - & $\uparrow 482300$ \\
\hline
\end{tabular}

Ainsi qu'il a été précédemment remarqué dans le cas de membranes organiques testées avec un module plan, le modèle décrit également très bien les performances de membranes minérales disposées selon une géométrie tubulaire. Cette souplesse d'utilisation est essentiellement due à la simplicité du modèle associée à l'estimation d'un seul paramètre.

\section{5 - CONCLUSIONS}

Tout en restant simple, le modèle proposé permet de rendre compte du transfert de matière à travers les membranes de nanofiltration. Ce modèle peut être prédictif si on connaît les paramètres structurels de la membrane, mais il peut également être utilisé pour caractériser les membranes dont on ne connaît 
pas les caractéristiques. Le modèle a ainsi été testé et validé dans le cas de membranes minérales et organiques pour des modules plans ou tubulaires avec des solutés non chargés. Ce travail est une première étape vers une étude plus vaste concernant la séparation de solutés chargés, sels ou petites molécules, où le partage est régi par un couplage entre l'effet stérique et une exclusion électrostatique.

\section{NOMENCLATURE}

$c_{s}$ : concentration dans le pore $\left(\mathrm{mol} \cdot \mathrm{m}^{-3}\right)$

$C_{m}$ : concentration à l'interface alimentation $/$ membrane $\left(\mathrm{mol} \cdot \mathrm{m}^{-3}\right)$

$C_{0}$ : concentration dans l'alimentation $\left(\mathrm{mol} \cdot \mathrm{m}^{-3}\right)$

$c_{p}$ : concentration du perméat $\left(\mathrm{mol} \cdot \mathrm{m}^{-3}\right)$

$d_{h}: \quad$ diamètre hydraulique $(\mathrm{m})$

$D_{\mathrm{s}}$ : coefficient de diffusion du soluté dans le pore $\left(\mathrm{m}^{2} \cdot \mathrm{s}^{-1}\right)$

$D_{\infty}$ : coefficient de diffusion du soluté dans la solution d'alimentation $\left(\mathrm{m}^{2} \cdot \mathrm{s}^{-1}\right)$

$J_{s}: \quad$ flux molaire du soluté $\left(\mathrm{mol} \cdot \mathrm{m}^{-2} \cdot \mathrm{s}^{-1}\right)$

$J_{v}: \quad$ densité de flux volumique $\left(\mathrm{m} \cdot \mathrm{s}^{-1}\right)$

$k$ : coefficient de transfert de matière $\left(\mathrm{m} \cdot \mathrm{s}^{-1}\right)$

$L$ : $\quad$ longueur du pore $(\mathrm{m})$

$r_{s}$ : rayon de Stokes du soluté $(m)$

$r_{p}$ : rayon moyen du pore $(\mathrm{m})$

$R e:$ nombre de Reynolds (-)

$R_{m}$ : taux de rétention intrinsèque $(-)$

$R_{\text {obs }}$ : taux de rétention observé (-)

Sc : nombre de Schmidt (-)

Sh: nombre de Sherwood (-)

$U$ : vitesse d'écoulement dans la cellule $\left(\mathrm{m} \cdot \mathrm{s}^{-1}\right)$

$V: \quad$ vitesse d'écoulement dans le pore $\left(\mathrm{m} \cdot \mathrm{s}^{-1}\right)$

$\alpha: \quad$ paramètre défini selon l'Eq.14 $\left(\mathrm{s} \cdot \mathrm{m}^{-1}\right)$

$\delta$ : épaisseur de la polarisation de concentration (m)

$\varepsilon$ : porosité $(-)$

$\Phi_{S}: \quad$ coefficient de partage stérique (-) 


\section{RÉFÉRENCES BIBLIOGRAPHIQUES}

BATICLE P., KIEFER C., LAKHCHAF N., LARBOT A., LECLERC O., PERSIN M., SARRAZIN J., 1997. Salt filtration on gamma alumina nanofiltration membranes fired at two different temperatures. J. Membrane Sci., 135, 1-8.

BERTRAN S., GAID A., PAILLARD H., 1997. Application de la nanofiltration dans le traitement d'une eau résiduaire de papeterie. Procédés à membranes : maítrise du cycle de l'eau dans l'industrie. Pollutec, Paris, France.

BOWEN W.R., MOHAMMAD A.W., HILAL N., 1997. Characterisation of nanofiltration membranes for predictive purposes use of salts, uncharged solutes and atomic force microcopy. J. Membrane Sci. 126, 91-105.

BRUN J.P., 1988. Procédés de séparation par membranes. Edts MASSON, Paris.

COMBE C., GUIZARD C., AIMAR P., SANCHEZ V., 1997. Experimental determination of four characteristics used to predict the retention of a ceramic nanofiltration membrane. J. Membrane Sci., $129,147-160$.

LEVENSTEIN R., HASSON D., SEMIAT R., 1996. Utilization of the Donnan effect for improving electrolyte separation with nanofiltration membranes,. J. Membrane Sci., 116, 77-92.

MEHIGUENE K., GARBA Y., TAHA S., GONDREXON N., DORANGE G., 1999. Influence of operating conditions on the retention of copper and cadmium in aqueous solutions by nanofiltration : experimental results and modelling. Sep. and Pur. Tech., 15, 181-187.

NAKAO S.I., KIMURA S., 1981. Analysis of solutes rejection in ultrafiltration. $J$. Chem. Eng. Jpn., 14, 32-37.

RIOS G.M., JOULIE R., SARRADE S.J., CARLES M., 1996. Investigation of ion separation by microporous nanofiltration membranes. AlChE J., 42(9), 2521-2528.

ROSA M.J., de PINHO M.N., 1994. Separation of organic solutes by membranes pressure-driven process. J. Membrane Sci., 89, 235-243.

SARRADE S., RIOS G M., CARLES M., 1994. Dynamic characterization and transport mechanisms of two inorganic membranes for nanofiltration. J. Membrane Sci. 97, 155-166.

SPIEGLER K.S., KEDEM O., 1966. Thermodynamic of hyperfiltration $(\mathrm{RO})$ criteria for efficient membranes. Desalination, 1, 311-326.

TIMMER J.M.K., KROMKAMP J., ROBBERTSEN T., 1994. Lactic acid separation from fermentation broths by reverse osmosis and nanofiltration. J. Membrane Sci. , 92, 185-197.

TSURU T., IZUMI S., YOSHIOKA T., MASASHI M., 1999. Nanofiltration performance of neutral solutes by inorganic porous membranes. International Congress on Membranes and Membranes Processes (ICOM), Toronto, 12-18.

Van der HORST H.C., TIMMER J.M.K., ROBBERTSEN T., LEENDERS J., 1995. Use of nanofiltration for concentration and demineralization in the dairy industry: model for mass transport. $J$. Membrane Sci., 104, 205-218.

WADLEY S., BROUCKAERT C.J., BADDOCK L.A.D., BUCKLEY C.A., 1995. Modelling of nanofiltration applied to the recovery of salt from waste brine at sugar decolourisation plant. J. Membrane Sci., 102, 163-175.

YAROSHCHUCK A.E, 1999. Rejection mechanisms of NF membranes: in what way can they be identified through advanced characterization of membrane transport properties. International Congress on Membranes and Membranes Processes (ICOM), Toronto.

YAROSHCHUCK A.E, VOVKOGON Y.A., 1994. Phenomenological theory of pressur-driven transport of ternary electrolyte solutions with a common coion and its specification for capillary space-charge model. J. Membrane Sci., 86, 1-18. 